\title{
Lymphocyte Cell Ratios and Mortality among Incident Hemodialysis Patients
}

\author{
Christina Catabay $^{\mathrm{a}}$ Yoshitsugu Obi $^{\mathrm{a}}$ Elani Streja ${ }^{\mathrm{a}, \mathrm{b}}$ Melissa Soohoo ${ }^{\mathrm{a}}$ \\ Christina Park $^{a}$ Connie M. Rhee ${ }^{a}$ Csaba P. Kovesdyc, ${ }^{c}$ Takayuki Hamano ${ }^{e}$ \\ Kamyar Kalantar-Zadeh ${ }^{a, b, f}$
}

\begin{abstract}
${ }^{a}$ Harold Simmons Center for Kidney Disease Research and Epidemiology, Division of Nephrology and Hypertension, University of California Irvine, School of Medicine, Orange, CA, USA; ${ }^{b}$ Nephrology Section, Tibor Rubin Veterans

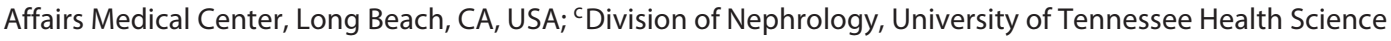
Center, Memphis, TN, USA; ${ }^{\mathrm{d}}$ Nephrology Section, Memphis VA Medical Center, Memphis, TN, USA; ${ }^{e}$ Department of Comprehensive Kidney Disease Research, Osaka University Graduate School of Medicine, Osaka, Japan; ${ }^{\mathrm{f}}$ Fielding School of Public Health at UCLA, Los Angeles, CA, USA
\end{abstract}

\section{Keywords \\ Hemodialysis - Chronic hemodialysis - End-stage kidney disease · Neutrophil · Platelet - Lymphocyte · Mortality . Nutrition · Inflammation}

\begin{abstract}
Background: Neutrophil-to-lymphocyte ratio (NLR) and platelet-to-lymphocyte ratio (PLR) have been previously suggested as oncologic prognostication markers. These are associated with malnutrition and inflammation, and hence, may provide benefit in predicting mortality among hemodialysis patients. Methods: Among 108,548 incident hemodialysis patients in a large U.S. dialysis organization (20072011), we compared the mortality predictability of NLR and PLR with baseline and time-varying covariate Cox models using the receiver operating characteristic curve (AUROC), net reclassification index (NRI), and adjusted $\mathrm{R}^{2}$. Results: During the median follow-up period of 1.4 years, 28,618 patients died. Median (IQR) NLR and PLR at baseline were 3.64 (2.68$5.00)$ and 179 (136-248) respectively. NLR was associated
\end{abstract}

๑ 2017 S. Karger AG, Basel with higher mortality, which appeared stronger in the timevarying versus baseline model. PLR exhibited a J-shaped association with mortality in both models. NLR provided better mortality prediction in addition to demographics, comorbidities, and serum albumin; $\triangle \mathrm{AUROC}$ and $\mathrm{NRI}$ for 1-year mortality $(95 \% \mathrm{Cl})$ were $0.010(0.009-0.012)$ and $6.4 \%(5.5-$ $7.3 \%)$ respectively. Additionally, adjusted $\mathrm{R}^{2}(95 \% \mathrm{CI})$ for the Cox model increased from $0.269(0.262-0.276)$ to 0.283 $(0.276-0.290)$ in the non-time-varying model and from 0.467 $(0.461-0.472)$ to $0.505(0.500-0.512)$ in the time-varying model. There was little to no benefit of adding PLR to predict mortality. Conclusions: High NLR in incident hemodialysis patients predicted mortality, especially in the short-term period. NLR, but not PLR, added modest benefit in predicting mortality along with demographics, comorbidities, and serum albumin, and should be included in prognostication approaches.

(c) 2017 S. Karger AG, Basel

\section{KARGER}

E-Mail karger@karger.com

www.karger.com/ajn
Kamyar Kalantar-Zadeh, MD, PhD, MPH and Yoshitsugu Obi, MD, PhD Harold Simmons Center for Kidney Disease Research and Epidemiology University of California Irvine, 333 City Blvd West, City Tower, Suite 400 Orange, CA 92868 (USA)

E-Mailkkz@uci.edu and yobi@uci.edu 


\section{Introduction}

Low-grade systemic and persistent inflammation may play an important role in the development and progression of various chronic conditions and diseases such as obesity, diabetes, atherosclerotic cardiovascular disease, cancer, chronic obstructive lung disease, and chronic kidney disease (CKD) [1-3]. Among patients with endstage renal disease (ESRD), chronic inflammation is highly prevalent and associated with adverse clinical outcomes including cardiovascular disease and all-cause death [4-8]. Inflammation in this context is attributed to various underlying factors including the uremic milieu, infections, decreased clearance of proinflammatory cytokines, volume overload, endotoxinemia, oxidative stress, carbonyl stress, and dialysis-related factors [912]. A recent study found increased arterial wall inflammation even in non-dialysis patients with CKD [13], which may explain the cardiovascular risk associated with CKD [14]. Inflammation can also interact with malnutrition and lead to wasting and derangements in protein - energy nutritional status, that is, protein-energy wasting, resulting in the excessively high mortality in the dialysis population $[10,12]$. C-reactive protein, interleukin-6, and tumor necrosis factor- $\alpha$ are among representative inflammatory markers, but to get them measured is an expensive task and/or they are inaccessible in current clinical practice.

The neutrophil-to-lymphocyte ratio (NLR) and platelet-to-lymphocyte ratio (PLR), readily available biomarkers calculated from complete blood count, are closely associated with inflammation and were originally suggested as oncologic prognostic factors $[15,16]$. Subsequently higher values in NLR and PLR have been linked to greater inflammatory status and worse prognosis among patients with various disease conditions including cardiovascular disease [17], chronic obstructive pulmonary disease [18], liver cirrhosis [19], and CKD [20]. Furthermore, recent studies have indicated that NLR and PLR were associated with inflammation and may predict mortality among hemodialysis patients as well [21-26], but their small study sample sizes make it difficult to draw a definite conclusion regarding their detailed associations with mortality and comparative usefulness in mortality-predictability when compared to other clinically accessible markers related to CKD. To address this knowledge gap, we studied a 5-year large national cohort of incident hemodialysis patients in the United States and investigated the relationship of NLR and PLR with all-cause mortality.

Lymphocyte Ratios in HD

\section{Methods}

This study was approved by the Institutional Review Committees of the Los Angeles Biomedical Research Institute at HarborUCLA, University of California Irvine Medical Center, and the University of Washington, with the exemption of obtaining written consent, given the large sample size, anonymity of the patients studied, and nonintrusive nature of the research.

\section{Patients}

We retrospectively extracted, refined, and examined data from all ESRD patients aged $\geq 18$ who initiated dialysis from January 1 , 2007 to December 31, 2011 and who received dialysis treatment in one of the facilities operated by a large dialysis organization in the United States [27]. Patients were followed up from the of first date dialysis until death, kidney transplantation, loss to follow-up, or the date of final follow-up assessment for all patients (i.e., December 31, 2011). The follow-up time was divided into patient-quarters (i.e., 91-day periods from the first date of dialysis).

Out of the 156,229 patients who were initially treated with conventional hemodialysis, we excluded 30,493 patients who did not receive dialysis treatment for $\geq 60$ consecutive days, 13,719 patients who were ever treated with other modalities (e.g., less frequent hemodialysis, frequent hemodialysis, nocturnal hemodialysis, home hemodialysis, or peritoneal dialysis), 2,593 and 579 patients with missing data and NLR values $>14$ (i.e., $>99.5$ percentile), respectively, on complete blood count markers. We also excluded 297 patients without data on serum albumin, phosphorus, and alkaline phosphatase (ALP) at baseline (Fig. 1).

\section{Demographic, Clinical, and Laboratory Measures}

Information on death, race/ethnicity, primary insurance, access type, the presence of comorbidities, and laboratory variables was obtained from the administrative electronic database of the dialysis provider. To minimize measurement variability, all repeated measures for each patient were averaged within each patientquarter and then used in all analyses. The averaged values during the first patient-quarter (the first 91-days of dialysis) served as baseline data.

Blood samples were drawn using uniform techniques in all dialysis clinics and were transported to the central laboratory in Deland, Florida, typically within $24 \mathrm{~h}$. All laboratory values were measured by automated and standardized methods. Most laboratory values were measured monthly with the exception of serum ferritin and intact parathyroid hormone $(\mathrm{PTH})$ that were measured at least quarterly. Hemoglobin was measured monthly in essentially all patients and weekly to bi-weekly in most patients. NLR was calculated using percent neutrophil divided by percent lymphocyte, whereas PLR was calculated using platelet count divided by lymphocyte count. Single-pool Kt/V (spKt/V) was calculated using urea kinetic modeling equations [28].

\section{Statistics}

Differences in baseline characteristics between 2 groups were compared by standardized differences, of which $0.8,0.5$, and 0.2 in absolute value were considered large, medium, and small differences, respectively, due to the relatively large sample size of this study $[29,30]$. NLR and PLR, the primary exposures of this study, were categorized into 6 groups $(<2.0,2.0-<3.0,3.0-<4.0,4.0-<5.0$, $5.0-<6.0$, and $\geq 6.0$; and $<100,100-<150,150-<200,200-<250$, 


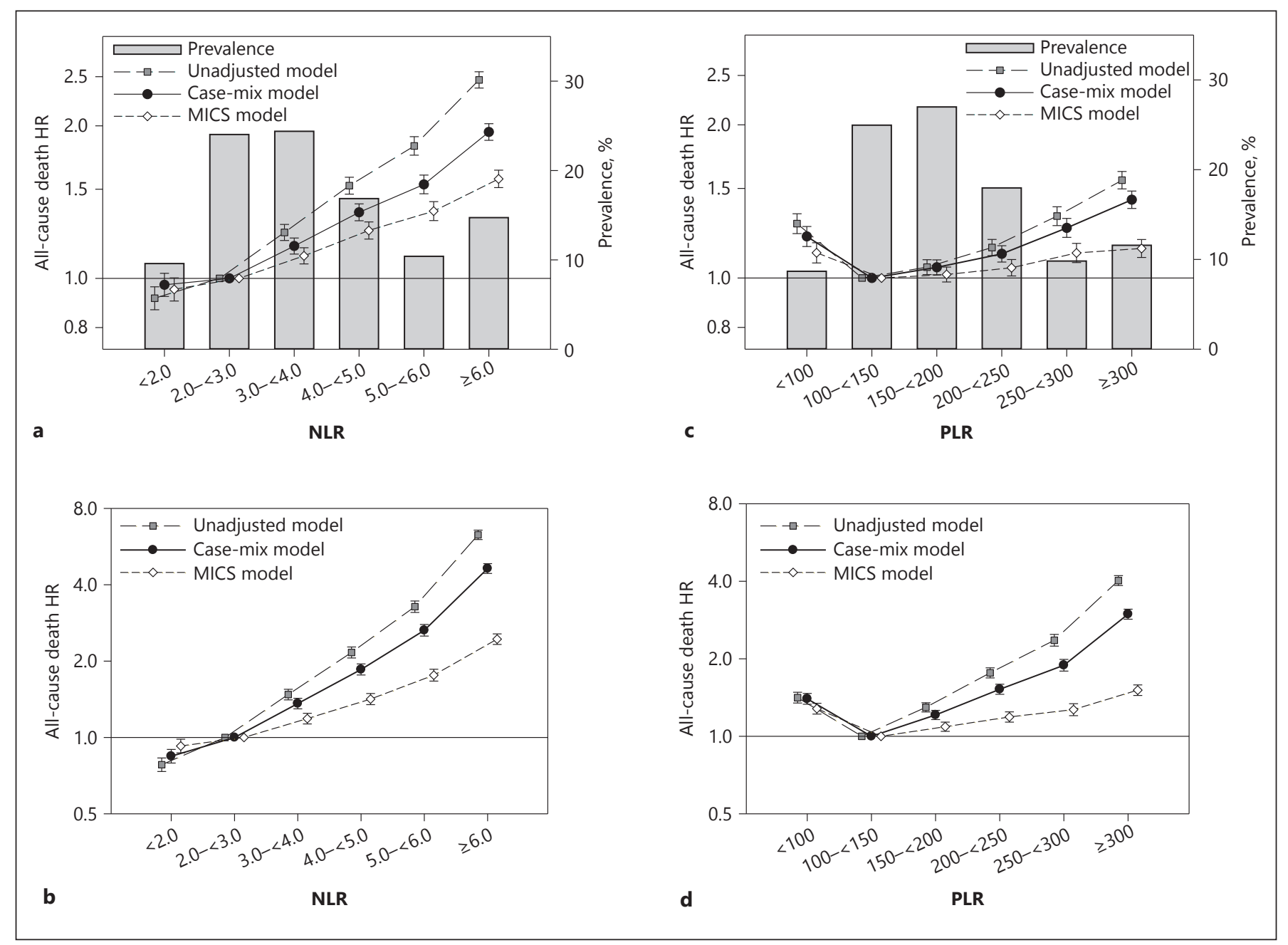

Fig. 1. Mortality risk associated with neutrophil-to-lymphocyte ratio (NLR) and platelet-to-lymphocyte ratio (PLR) after initiating dialysis among 108,548 incident hemodialysis patients (2007-2011) in the (a, c) baseline and $(\mathbf{b}, \mathbf{d})$ time-varying Cox models with 3-level hierarchical adjustments respectively.

$250-<300$, and $\geq 300$, respectively). Their associations with each patient characteristic were evaluated using a non-parametric trend test. Logistic regression was used to identify the predictors of high NLR or PLR using arbitrary cut offs (i.e., $\geq 4.0$ and $\geq 200$, respectively). Cox regression models with baseline (non-time-varying) or time-varying covariates were used to estimate associations with all-cause mortality. Models were examined with 3 levels of adjustment as follows:

I. Unadjusted model that included NLR or PLR;

II. Case-mix adjusted models that included the above parameters plus age, gender, race and ethnicity, primary insurance, 12 comorbidities as shown in Table 1, vascular access type, body mass index (BMI), and spKt/V; and

III. Malnutrition-inflammation-cachexia complex syndrome (MICS) adjusted models, which included all of the covariates in the case-mix model plus 9 laboratory variables related to the MICS (i.e., white blood cell [WBC] count, hemoglobin, serum albumin, creatinine, albumin-corrected calcium, phosphorus, iron satura- tion, total bicarbonate, ALP, total iron binding capacity [TIBC], and natural log-transformed intact PTH and ferritin).

Time-varying covariates included vascular access type, BMI, $\mathrm{spKt} / \mathrm{V}$, and laboratory variables. In order to compare mortalitypredictabilities of NLR and PLR with those of albumin and ALP, the mortality risk was estimated according to the deciles of each variable. The reason for selecting serum albumin and ALP for the comparison is that both indices have been consistently and linearly associated with mortality among hemodialysis patients [31, 32]. Serum albumin is a representative MICS marker, while ALP is not in the realm of MICS but is an abnormality observed in ESRD as a part of CKD-MBD. The area under the receiver operating characteristic curve (AUROC) and net reclassification index (NRI) was examined for 1-year mortality using logistic regression with different sets of variables at baseline. Additionally, explained variation (i.e., adjusted $\mathrm{R}^{2}$, a transformation of Royston and Sauerbrei's D measure of discrimination of a survival model) [33] was evaluated in both time-varying and non-time-varying Cox models 
Table 1. Baseline demographic and clinical characteristics of 108,548 incident hemodialysis patients

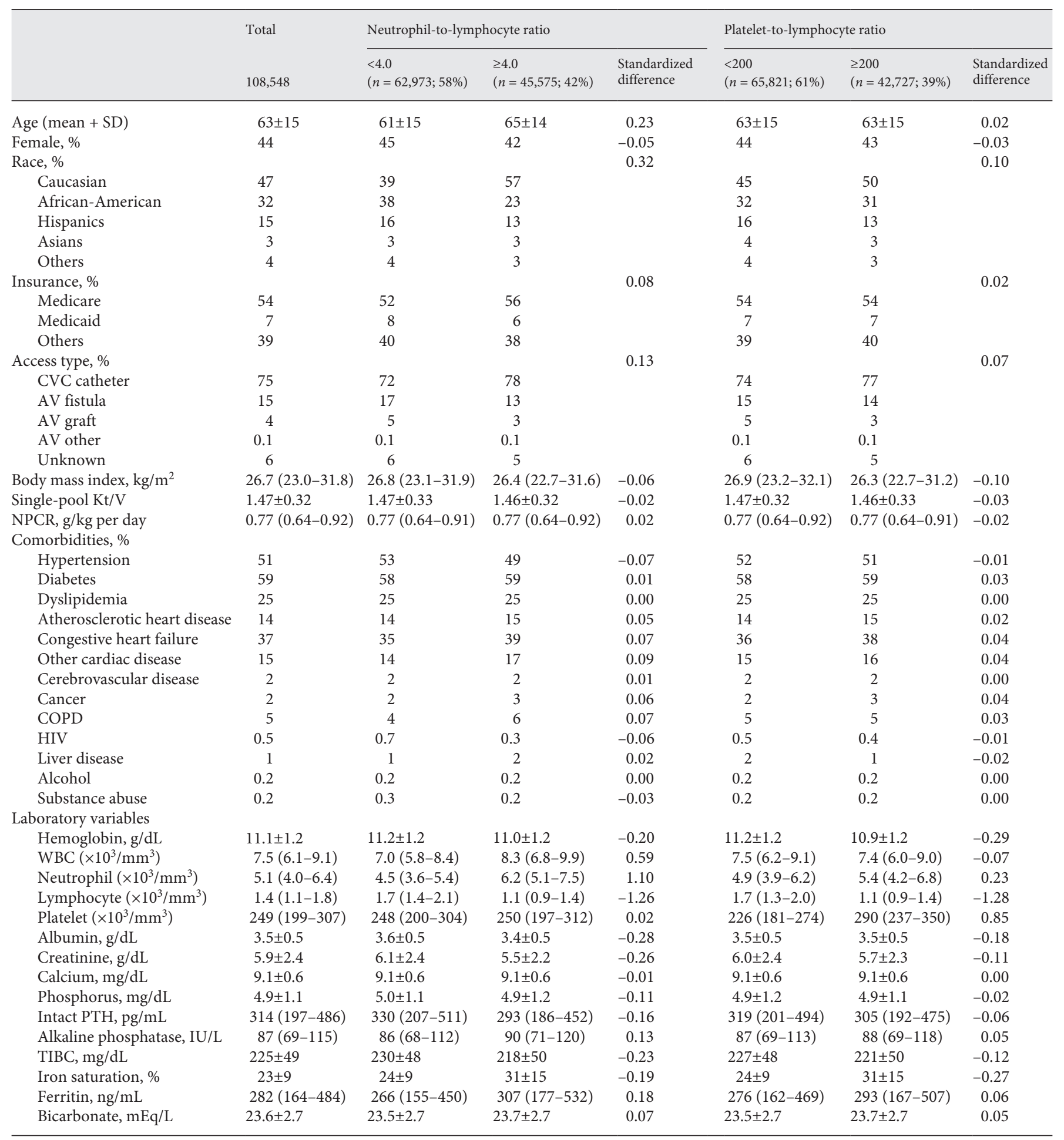

Values are expressed as mean \pm SD, median (IQR), or percentage, appropriately. Differences in patient characteristics between two groups were compared by standardized difference, of which $0.8,0.5$, and 0.2 in absolute value were considered large, medium, and small differences respectively. COPD, chronic obstructive pulmonary disease; HIV, human immunodeficiency virus; AIDS, acquired immune deficiency syndrome; WBC, white blood cell; PTH, parathyroid hormone; TIBC, total iron binding capacity. Conversion factors for units: albumin and hemoglobin in $\mathrm{g} / \mathrm{dL}$ to $\mathrm{g} / \mathrm{L}, 10$; creatinine in $\mathrm{mg} / \mathrm{dL}$ to $\mathrm{mmol} / \mathrm{L}, 88.4$; calcium in $\mathrm{mg} / \mathrm{dL}$ to $\mathrm{mmol} / \mathrm{L}, 0.2495$; phosphorus in $\mathrm{mg} / \mathrm{dL}$ to $\mathrm{mmol} / \mathrm{L}, 0.3229$. No conversion is necessary for ferritin in $\mathrm{ng} / \mathrm{mL}$ and $\mathrm{mg} / \mathrm{L}$. 
to compare the overall performance measure across models. The frequency of missing data was low ( $<1 \%$ for BMI and most laboratory tests, except for ferritin [1.2\%], nPCR [1.5\%], and creatinine $[4.6 \%])$, and mean imputation was used in all regression analyses. All analyses were conducted using STATA MP version 13.1 (StataCorp, College Station, TX, USA).

\section{Results}

\section{Baseline Demographic, Clinical, and Laboratory Characteristics}

Baseline characteristics were compared between included and excluded patients and we found that the 108,548 included patients were less likely to be Caucasian; and were more likely to have a CV catheter as their initial vascular access. Compared to the 47,681 excluded patients who lacked NLR or PLR data, included patients had higher hemoglobin, lower WBC count, lower neutrophil, higher lymphocyte, higher albumin, higher creatinine, higher phosphorus, higher intact $\mathrm{PTH}$, higher TIBC, lower ferritin, lower bicarbonate, and higher prevalence of hypertension, diabetes, and congestive heart failure at baseline (online suppl. Table 1; for all online suppl. material, see www.karger.com/ doi/10.1159/000484177). The mean \pm SD age of the final study cohort was $63 \pm 15$ of whom $56 \%$ were male, $47 \%$ were Caucasian, $15 \%$ were Hispanic, and $59 \%$ were diabetic (Table 1). The median (interquartile range [IQR]) of NLR and PLR were 3.64 (2.68-5.00) and 179 (136248 ) respectively. As NLR values increased, the absolute lymphocyte count decreased, while the absolute counts of WBC and neutrophil increased. The absolute platelet count values were not different between the high and low NLR groups. Patients with higher NLR values were more likely to be older and Caucasian, but less likely to be African American. Likewise, as PLR values increased, the absolute lymphocyte count decreased, while the absolute neutrophil and platelets counts increased. There were no meaningful differences in demographics, comorbid conditions, and the absolute WBC count between the high and low NLR groups.

\section{Biochemical Associations of NLR and PLR}

After adjustment for case-mix variables, baseline laboratory variables associated with higher NLR (vs. below the median) included lower levels of hemoglobin, albumin, creatinine, albumin-corrected calcium, phosphorus, intact PTH, TIBC, and iron saturation and higher levels of WBC, ALP, ferritin, and bicarbonate (online suppl. Table 4). PLR exhibited similar association patterns for those laboratory variables except for WBC, where lower levels of WBC were associated with higher PLR. Calcium and phosphorus levels were not associated with PLR.

\section{Associations of NLR and PLR with All-Cause Mortality}

Out of 108,548 patients, a total of 28,618 patients died during the median follow-up period of 1.4 years (IQR, 0.6-2.5 years) with an incidence rate of 15.6 (95\% CI $15.4-15.7)$ per 100 patient-years. Baseline characteristics of the study cohort stratified by 6 NLR groups and 6 PLR groups are presented in online supplemental Tables 2, 3 respectively. Unadjusted non-time-varying (baseline) Cox models demonstrated that higher NLR levels were linearly associated with all-cause mortality risk. Those who had greater NLR experienced incrementally higher mortality, which was robust against adjustments for case-mix and MICS characteristics ( $p_{\text {trend }}<0.001$ for all; Fig. 1a and online suppl. Table 5). Similarly, the timevarying covariate model demonstrated incremental mortality risk across greater NLR irrespective of adjustment model, which appeared stronger than those observed in the baseline model ( $p_{\text {trend }}<0.001$ for all; Fig. $1 b$ ). When compared to NLR in the range $2.0-<3.0$, a hazard ratio (HR) of $\geq 6.0$ NLR was 1.94 (95\% CI 1.87-2.02) and 4.54 (95\% CI 4.34-4.75) in the baseline vs. the time-varying covariate Cox model with adjustment for case-mix variables respectively.

Baseline PLR exhibited a J-shaped association with allcause mortality in all adjustment models; the lowest risk was observed in the second lowest group (i.e., PLR 100$<150$ ), and higher levels were associated with all-cause mortality risk (Fig. 1c and online suppl. Table 5). As with NLR, the time-varying covariate model exhibited a consistent yet stronger association between PLR and allcause mortality when compared to the baseline model (Fig. 1d). Using PLR of 100-150 as reference, a case-mix adjusted HR of $\geq 300$ PLR was 1.43 (95\% CI $1.37-1.48$ ) and 2.95 (95\% CI 2.82-3.09) in the baseline vs. time-varying covariate Cox model respectively.

\section{Mortality Predictability Comparisons of Selected Laboratory Variables}

In order to evaluate the clinical usefulness in predicting mortality, we compared the HRs (Fig. 2) of deciles among serum albumin, serum ALP, NLR, and PLR, and then compared AUROC (Table 2), NRI (Table 2 and online suppl. Tables 6-12), and adjusted $\mathrm{R}^{2}$ (Table 3 ) among lymphocyte, neutrophil, and platelet counts in addition to those variables mentioned above. 


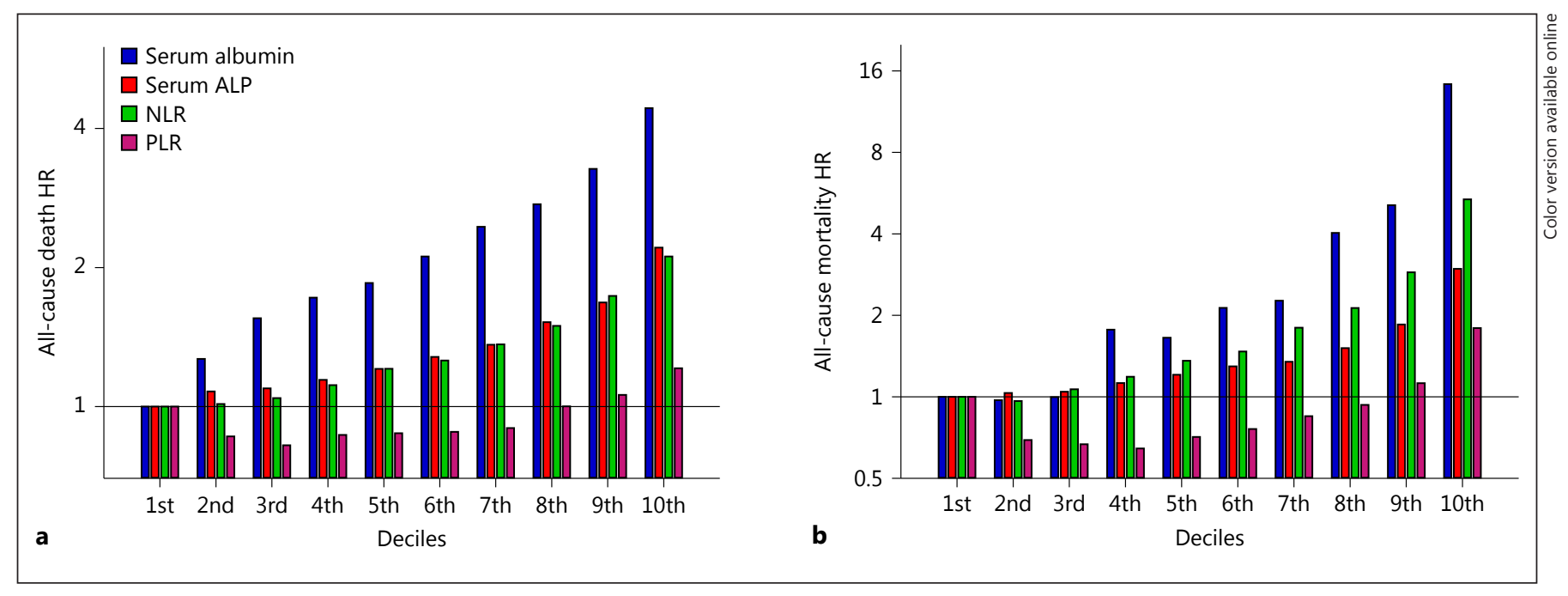

Fig. 2. Case-mix adjusted all-cause mortality HRs of deciles of (a) baseline and (b) time-varying serum albumin, serum alkaline phosphatase (ALP), neutrophil-to-lymphocyte ratio (NLR), and platelet-to-lymphocyte ratio (PLR) among 108,548 patients. Only

the deciles of serum albumin were ordered in reverse (i.e., the highest on the left and the lowest on the right) for the comparison with the other indices.

Table 2. Area under receiver operating characteristic curve (AUROC) and net reclassification index (NRI) for 1-year mortality by adding baseline levels of serum albumin, serum alkaline phosphatase (ALP), lymphocyte, neutrophil, platelet, neutrophil-to-lymphocyte ratio (NLR), and platelet-to-lymphocyte ratio (PLR) in addition to the case-mix variables

\begin{tabular}{lllc}
\hline & AUROC & AAUROC & NRI, \% \\
\hline Case-mix variables & $0.701(0.696-0.705)$ & Reference & Reference \\
Plus serum albumin & $0.743(0.738-0.747)$ & $0.042(0.039-0.045)$ & $16.1(14.9-17.2)$ \\
Plus serum alkaline phosphatase & $0.713(0.709-0.717)$ & $0.012(0.011-0.014)$ & $5.8(5.0-6.5)$ \\
Plus lymphocyte & $0.706(0.702-0.710)$ & $0.005(0.004-0.007)$ & $5.4(4.6-6.2)$ \\
Plus neutrophil & $0.708(0.704-0.713)$ & $0.008(0.006-0.009)$ & $5.6(4.7-6.5)$ \\
Plus platelet & $0.702(0.697-0.706)$ & $0.001(0.000-0.002)$ & $1.6(1.0-2.1)$ \\
Plus neutrophil and lymphocyte & $0.716(0.712-0.721)$ & $0.016(0.014-0.018)$ & $9.3(8.3-10.3)$ \\
Plus platelet and lymphocyte & $0.706(0.702-0.710)$ & $0.005(0.004-0.007)$ & $5.2(4.4-6.1)$ \\
Plus NLR & $0.719(0.715-0.724)$ & $0.019(0.017-0.021)$ & $9.5(8.5-10.5)$ \\
Plus PLR & $0.703(0.699-0.707)$ & $0.002(0.002-0.003)$ & $2.4(1.8-3.1)$ \\
Case-mix variables + serum albumin & See above & See above & See above \\
Plus serum alkaline phosphatase & $0.747(0.743-0.751)$ & $0.004(0.004-0.005)$ & $2.8(2.3-3.4)$ \\
Plus lymphocyte & $0.747(0.743-0.751)$ & $0.004(0.003-0.005)$ & $4.1(3.3-4.8)$ \\
Plus neutrophil & $0.745(0.741-0.749)$ & $0.002(0.002-0.003)$ & $2.9(2.2-3.6)$ \\
Plus platelet & $0.744(0.740-0.749)$ & $0.002(0.001-0.002)$ & $1.9(1.3-2.6)$ \\
Plus neutrophil and lymphocyte & $0.751(0.747-0.755)$ & $0.008(0.007-0.010)$ & $6.3(5.5-7.2)$ \\
Plus platelet and lymphocyte & $0.748(0.743-0.752)$ & $0.005(0.004-0.006)$ & $4.8(4.0-5.5)$ \\
Plus NLR & $0.753(0.749-0.757)$ & $0.010(0.009-0.012)$ & $6.4(5.5-7.3)$ \\
Plus PLR & $0.744(0.740-0.748)$ & $0.001(0.001-0.001)$ & $1.0(0.6-1.5)$ \\
\hline
\end{tabular}

Serum albumin appeared to be the strongest predictor of all-cause mortality in both the non-time-varying and time-varying Cox models with adjustment for the case-mix variables (Fig. 2), with the greatest AUROC and the highest NRI for 1-year mortality (Table 2). Adjusted $R^{2}$ of serum albumin was also the highest in both the non-time-varying and time-varying Cox model (Table 3). Baseline serum ALP and neutrophil, when compared to lymphocyte and platelet, showed higher AUROC, NRI, and adjusted $\mathrm{R}^{2}$, and neutrophil showed a higher adjusted $\mathrm{R}^{2}$ than serum 
Table 3. Adjusted $\mathrm{R}^{2}$ in the non-time-varying and time-varying Cox models by adding serum albumin, serum alkaline phosphatase (ALP), lymphocyte, neutrophil, platelet, neutrophil-to-lymphocyte ratio (NLR), and platelet-to-lymphocyte ratio (PLR) in addition to the case-mix variables

\begin{tabular}{lll}
\hline & Non-time-varying model & Time-varying model \\
\hline Case-mix variables & $0.203(0.196-0.210)$ & $0.307(0.302-0.315)$ \\
Plus serum Alb & $0.269(0.263-0.277)$ & $0.484(0.479-0.491)$ \\
Plus ALP & $0.218(0.210-0.225)$ & $0.329(0.324-0.336)$ \\
Plus lymphocyte & $0.211(0.204-0.216)$ & $0.331(0.326-0.337)$ \\
Plus neutrophil & $0.218(0.213-0.226)$ & $0.379(0.373-0.385)$ \\
Plus platelet & $0.204(0.196-0.210)$ & $0.308(0.302-0.315)$ \\
Plus neutrophil and lymphocyte & $0.229(0.222-0.236)$ & $0.406(0.401-0.411)$ \\
Plus platelet and lymphocyte & $0.211(0.205-0.217)$ & $0.335(0.329-0.341)$ \\
Plus NLR & $0.230(0.224-0.237)$ & $0.398(0.393-0.403)$ \\
Plus PLR & $0.207(0.201-0.213)$ & $0.333(0.328-0.340)$ \\
Case-mix variables + serum Alb & & $0.487(0.482-0.493)$ \\
Plus ALP & $0.278(0.272-0.287)$ & $0.490(0.485-0.497)$ \\
Plus Lymphocyte & $0.276(0.270-0.282)$ & $0.503(0.500-0.509)$ \\
Plus neutrophil & $0.276(0.270-0.284)$ & $0.484(0.480-0.489)$ \\
Plus platelet & $0.273(0.267-0.280)$ & $0.513(0.507-0.518)$ \\
Plus neutrophil and lymphocyte & $0.284(0.276-0.292)$ & $0.490(0.486-0.496)$ \\
Plus platelet and lymphocyte & $0.277(0.271-0.286)$ & $0.510(0.504-0.516)$ \\
Plus NLR & $0.286(0.279-0.293)$ & $0.488(0.483-0.493)$ \\
Plus PLR & $0.270(0.261-0.278)$ &
\end{tabular}

ALP in the time-varying model. However, NLR and the combination of neutrophil and lymphocyte demonstrated equivalent mortality predictability, which was better than serum ALP especially in the time-varying model. Neither PLR nor the combination of platelet and lymphocyte provided clinically relevant gain in these indices. When baseline NLR was added into the model including the case-mix variables and serum albumin, $\triangle$ AUROC and NRI were 0.010 (95\% CI 0.009-0.012) and 6.4\% (95\% CI 5.5-7.3\%), respectively, which was greater than those observed with serum ALP and PLR. It also increased adjusted $\mathrm{R}^{2}$ from $0.269(0.263,0.277)$ to $0.286(0.279,0.293)$ in the non-timevarying model and from $0.484(0.479,0.491)$ to 0.510 $(0.504,0.516)$ in the time-varying model.

Sensitivity analysis including patients with baseline NLR values $>14$ (i.e., $>99.5$ percentile) showed consistent results with a slight attenuation in the mortality predictability of NLR (data not shown)

\section{Discussion}

We demonstrated that high NLR was a strong predictor of all-cause death among hemodialysis patients. Patients with malnutrition and inflammation were likely to have high NLR. Baseline NLR was incrementally associ- ated with higher mortality and provided modest benefit to mortality prediction in addition to the case-mix variables and serum albumin. The NLR-mortality association was more pronounced in the time-varying model, suggesting a stronger association of NLR with short-term mortality than with long-term mortality. The mortality predictability of NLR appeared to be similar to or better than that of serum ALP or the combination of neutrophil and lymphocyte. Although PLR was associated with MICS variables, it exhibited a J-shaped association with mortality, and there was little, if any, improvement in mortality-predictability by adding either PLR or the combination of platelet and lymphocyte to the model including the case-mix variables and serum albumin.

Previous studies of dialysis patients demonstrated that both NLR and PLR had moderate correlations with direct inflammatory markers such as C-reactive protein, interleukin- 6 , and tumor necrosis factor- $\alpha[21,26,34]$ and that higher NLR and PLR values were associated with greater mortality $[23,25]$. Some studies also suggested that PLR may have a stronger correlation with inflammation and better mortality-predictability than NLR $[25,26]$. However, these studies had small sample sizes ( $<200$ patients), and hence, were susceptible to over-adjustment in multivariable analyses. Additionally, it remained difficult to judge the benefit of adding NLR or PLR as a new predic- 
tor to an existing model because these studies did not evaluate performance measures such as AUROC, NRI, and adjusted $\mathrm{R}^{2}$. The large sample size of our cohort allowed us to rigorously adjust for clinically relevant factors and to evaluate several model performances, all of which supported the better mortality-predictability of NLR than PLR.

Serum albumin has good correlations with NLR [34] because inflammatory status leads to high NLR by increasing neutrophils and decreasing lymphocytes, and also because it results in low serum albumin levels by downregulating albumin gene transcription [35-38]. Additionally, both serum albumin and lymphocyte count have been used as indices of nutritional status. Thus, serum albumin and NLR share common pathophysiological rationale for survival prediction. However, a previous study highlighted the lack of correlation between total lymphocyte count and nutritional status in the elderly [39], while recent randomized clinical trials demonstrated that protein supplementation increased serum albumin levels, which may explain the stronger association of serum albumin with survival than NLR in our study. Nevertheless, NLR modestly improved mortality prediction in addition to the case-mix variables and serum albumin. This additional benefit of adding NLR might be related to its shorter time to response to inflammation than serum albumin; neutrophils are rapidly released from the bone marrow into the bloodstream with a short half-life $(\sim 6-$ $8 \mathrm{~h})$ [40], whereas serum albumin decreases through reduced synthesis rate and has a longer half-life $(\sim 19-21$ days).

We acknowledge several limitations in this study. First, due to the observational nature of our study, we cannot prove causality between NLR or PLR and death, nor exclude the possibility of residual confounding. Second, the administrative database from a large dialysis organization does not enable us to individually identify specific condition(s) for high NLR or PLR, such as infection, malnutrition, central venous catheter, stress, metabolic syndrome, and atherosclerosis. Third, there are currently no established reference ranges for NLR and PLR in the general population or the dialysis population although a previous study of healthy individuals reported lower NLR values (2.5-97.5th percentiles, 0.8-3.5) [41] than those in our study. However, our survival analyses showed a linear association between higher NLR and higher mortality without clear thresholds indicating its optimal ranges. Finally, we included exclusively incident hemodialysis patients, and it remains unclear whether our findings can be extrapolated to prevalent hemodialysis patients or peritoneal di- alysis patients. Nevertheless, our study is the largest study of hemodialysis patients evaluating model performance measures of NLR and PLR, and both non-time-varying and time-varying models yielded consistent results.

In conclusion, a high NLR in incident HD patients predicted mortality, especially in the short-term period, and provided modest benefit to predict mortality in addition to demographics, comorbidities, and serum albumin. PLR demonstrated a weaker association with mortality, and there was small or virtually no gain in mortality-predictability. NLR, together with serum albumin, may be useful as a surrogate of nutritional and inflammatory status in both clinical and epidemiological research settings, given that it is readily available without additional cost.

\section{Acknowledgments}

This study is based on data provided by DaVita Clinical Research. The manuscript has been reviewed and approved by DaVita. The interpretation and conclusions are those of the authors and do not represent the views of DaVita.

The work in this manuscript has been performed with the support of the National Institute of Diabetes, Digestive and Kidney Disease (NIDDK) of the National Institute of Health research grants R01-DK95668 (KKZ), K24-DK091419 (KKZ), and R01DK078106 (KKZ). K.K.Z. is supported by philanthropic grants from Mr. Harold Simmons, Mr. Louis Chang, Dr. Joseph Lee and AVEO. YO is supported by the Uehara Memorial Foundation Research Fellowship. CPK is supported by the NIDDK grants R01DK096920 and U01-DK102163. CMR is supposed by the NIDDK grant K23-DK102903. ES is supposed by the VA Career Development Award IK2-CX 001266-01.

\section{Disclosure Statement}

K.K.Z. has received honoraria and/or support from Abbott, Abbvie, Alexion, Amgen, American Society of Nephrology, AstraZeneca, Aveo, Chugai, DaVita, Fresenius, Genentech, Haymarket Media, Hospira, Kabi, Keryx, National Institutes of Health, National Kidney Foundation, Relypsa, Resverlogix, Sanofi, Shire, Vifor, and ZS-Pharma.

References

Am J Nephrol 2017;46:408-416

1 Manabe I: Chronic inflammation links cardiovascular, metabolic and renal diseases. Circ J 2011;75:2739-2748.

2 Scrivo R, Vasile M, Bartosiewicz I, Valesini G: Inflammation as "common soil" of the multifactorial diseases. Autoimmun Rev 2011;10: 369-374.

3 Fabbri LM, Rabe KF: From COPD to chronic systemic inflammatory syndrome? Lancet 2007;370:797-799. 
4 Yeun JY, Levine RA, Mantadilok V, Kaysen GA: C-Reactive protein predicts all-cause and cardiovascular mortality in hemodialysis patients. Am J Kidney Dis 2000;35:469-476.

5 Owen WF, Lowrie EG: C-reactive protein as an outcome predictor for maintenance hemodialysis patients. Kidney Int 1998;54:627636.

6 Zimmermann J, Herrlinger S, Pruy A, Metzger T, Wanner C: Inflammation enhances cardiovascular risk and mortality in hemodialysis patients. Kidney Int 1999;55:648-658.

7 Iseki K, Tozawa M, Yoshi S, Fukiyama K: Serum C-reactive protein (CRP) and risk of death in chronic dialysis patients. Nephrol Dial Transplant 1999;14:1956-1960.

8 Noh H, Lee SW, Kang SW, et al: Serum Creactive protein: a predictor of mortality in continuous ambulatory peritoneal dialysis patients. Perit Dial Int 1998;18:387-394.

9 Hung SC, Lai YS, Kuo KL, Tarng DC: Volume overload and adverse outcomes in chronic kidney disease: clinical observational and animal studies. J Am Heart Assoc 2015;4:pii: e001918.

10 Kalantar-Zadeh K, Ikizler TA, Block G, Avram MM, Kopple JD: Malnutrition-inflammation complex syndrome in dialysis patients: causes and consequences. Am J Kidney Dis 2003;42:864-881.

11 Sarnak MJ: Cardiovascular complications in chronic kidney disease. Am J Kidney Dis 2003;41:11-17.

12 Obi Y, Qader H, Kovesdy CP, Kalantar-Zadeh $\mathrm{K}$ : Latest consensus and update on protein-energy wasting in chronic kidney disease. Curr Opin Clin Nutr Metab Care 2015;18:254-262.

13 Bernelot Moens SJ, Verweij SL, van der Valk FM, et al: Arterial and cellular inflammation in patients with CKD. J Am Soc Nephrol 2017; 28:1278-1285.

14 Go AS, Chertow GM, Fan D, McCulloch CE, Hsu C: Chronic kidney disease and the risks of death, cardiovascular events, and hospitalization. N Engl J Med 2004;351:1296-1305.

15 Li MX, Liu XM, Zhang XF, et al: Prognostic role of neutrophil-to-lymphocyte ratio in colorectal cancer: a systematic review and meta-analysis. Int J Cancer 2014;134:2403-2413.

16 Templeton AJ, McNamara MG, Eruga B, et al: Prognostic role of neutrophil-to-lymphocyte ratio in solid tumors: a systematic review and meta-analysis. J Natl Cancer Inst 2014; 106:dju124.

17 Bhat T, Teli S, Rijal J, et al: Neutrophil to lymphocyte ratio and cardiovascular diseases: a review. Expert Rev Cardiovasc Ther 2013;11: 55-59.

18 Günay E, Sarınç Ulaşlı S, Akar O, et al: Neutrophil-to-lymphocyte ratio in chronic obstructive pulmonary disease: a retrospective study. Inflammation 2014;37:374-380.

19 Biyik M, Ucar R, Solak Y, et al: Blood neutrophil-to-lymphocyte ratio independently predicts survival in patients with liver cirrhosis. Eur J Gastroenterol Hepatol 2013;25:435441.

20 Azab B, Daoud J, Naeem F Ben, et al: Neutrophil-to-lymphocyte ratio as a predictor of worsening renal function in diabetic patients (3-year follow-up study). Ren Fail 2012;34: 571-576.

21 Ahbap E, Sakaci T, Kara E, et al: Neutrophilto-lymphocyte ratio and platelet-tolymphocyte ratio in evaluation of inflammation in end-stage renal disease. Clin Nephrol 2016; 85:199-208.

22 Turkmen K, Guney I, Yerlikaya FH, Tonbul HZ: The relationship between neutrophil-tolymphocyte ratio and inflammation in endstage renal disease patients. Ren Fail 2012;34: 155-159.

23 Neuen BL, Leather N, Greenwood AM, Gunnarsson R, Cho Y, Mantha ML: Neutrophillymphocyte ratio predicts cardiovascular and all-cause mortality in hemodialysis patients. Ren Fail 2016;38:70-76.

24 Turkmen K, Ozcicek F, Ozcicek A, Akbas EM, Erdur FM, Tonbul HZ: The relationship between neutrophil-to-lymphocyte ratio and vascular calcification in end-stage renal disease patients. Hemodial Int 2014; 18:47-53.

25 Yaprak M, Turan MN, Dayanan R, et al: Platelet-to-lymphocyte ratio predicts mortality better than neutrophil-to-lymphocyte ratio in hemodialysis patients. Int Urol Nephrol 2016; 48:1343-1348.

26 Turkmen K, Erdur FM, Ozcicek F, et al: Platelet-to-lymphocyte ratio better predicts inflammation than neutrophil-to-lymphocyte ratio in end-stage renal disease patients. Hemodial Int 2013;17:391-396.

27 Kuttykrishnan S, Kalantar-Zadeh K, Arah OA, et al: Predictors of treatment with dialysis modalities in observational studies for comparative effectiveness research. Nephrol Dial Transplant 2015;30:1208-1217.

28 Daugirdas JT, Leypoldt JK, Akonur A, Greene T, Depner TA; FHN Trial Group: Improved equation for estimating single-pool Kt/V at higher dialysis frequencies. Nephrol Dial Transplant 2013;28:2156-2160.
29 Austin PC: Balance diagnostics for comparing the distribution of baseline covariates between treatment groups in propensity-score matched samples. Stat Med 2009;28:30833107.

30 Schacht A, Bogaerts K, Bluhmki E, Lesaffre E: A New nonparametric approach for baseline covariate adjustment for two-group comparative studies. Biometrics 2008;64:1110-1116.

31 Kalantar-Zadeh K, Kilpatrick RD, Kuwae N, et al: Revisiting mortality predictability of serum albumin in the dialysis population: time dependency, longitudinal changes and population-attributable fraction. Nephrol Dial Transplant 2005;20:1880-1888.

32 Regidor DL, Kovesdy CP, Mehrotra R, et al: Serum alkaline phosphatase predicts mortality among maintenance hemodialysis patients. J Am Soc Nephrol 2008;19:2193-2203.

33 Royston P: Explained variation for survival models. Stata J 2006;6:83-96.

34 Okyay GU, Inal S, Öneç K, et al: Neutrophil to lymphocyte ratio in evaluation of inflammation in patients with chronic kidney disease. Ren Fail 2013;35:29-36.

35 Birch HE, Schreiber G: Transcriptional regulation of plasma protein synthesis during inflammation. J Biol Chem 1986;261:80778080.

36 Princen JM, Nieuwenhuizen W, Mol-Backx GP, Yap SH: Direct evidence of transcriptional control of fibrinogen and albumin synthesis in rat liver during the acute phase response. Biochem Biophys Res Commun 1981;102: 717-723

37 Kaysen GA, Dubin JA, Müller HG, Mitch WE, Rosales LM, Levin NW: Relationships among inflammation nutrition and physiologic mechanisms establishing albumin levels in hemodialysis patients. Kidney Int 2002;61: 2240-2249.

38 Kaysen GA, Dubin JA, Müller HG, et al: Inflammation and reduced albumin synthesis associated with stable decline in serum albumin in hemodialysis patients. Kidney Int 2004:65:1408-1415.

39 Kuzuya M, Kanda S, Koike T, Suzuki Y, Iguchi A: Lack of correlation between total lymphocyte count and nutritional status in the elderly. Clin Nutr. 2005;24:427-432.

40 Sadik CD, Kim ND, Luster AD: Neutrophils cascading their way to inflammation. Trends Immunol 2011:32:452-460.

41 Forget $\mathrm{P}$, Khalifa C, Defour JP, et al: What is the normal value of the neutrophil-to-lymphocyte ratio? BMC Res Notes 2017;10:12. 